\title{
The Carathéodory-Féjer Interpolation Problem in Hardy Classes
}

\author{
D. Alpay and V. Bolotnikov
}

\begin{abstract}
In this paper we study the Carathéodory-Féjer interpolation problem in the Hardy spaces $\mathbf{H}_{2}$ of the unit disk. The results are presented within a framework which slightly generalizes the $\mathbf{H}_{2}$ spaces, and allow to consider in a unified way Hardy spaces of the unit disk and of a half plane.
\end{abstract}

Keywords: Carathéodory-Fejer interpolation, fundamental matrix inequalities

AMS subject classification: Primary 47A57, secondary 30E05

\section{Introduction}

We denote by $\mathbf{H}_{2}$ the Hardy spaces of functions analytic in the open unit disk $\mathbb{D}$ (see $[9,10])$. The Carathéodory-Féjer problem for $\mathbf{H}_{2}$ functions consists of the following:

Given $\omega \in \mathbb{D}$ and $h_{0}, \ldots, h_{N} \in \boldsymbol{C}$, find necessary and sufficient conditions for a function $h \in \mathbf{H}_{2}$ to exist such that

$$
\text { (i) }\|h\|_{\mathrm{H}_{2}} \leq 1 \quad \text { and } \quad\left(\text { ii) } h^{(k)}(\omega)=h_{k} \quad(k=0, \ldots, N)\right. \text {. }
$$

Since $\mathbf{H}_{2}$ is a reproducing kernel Hilbert space, this problem is easily solved using reproducing kernel Hilbert space methods, as we now briefly recall. We set $k(z, w)=$ $\frac{1}{1-z \bar{\omega}}$ to be the reproducing kernel of $\mathbf{H}_{2}$ and let

$$
\varphi_{\omega}^{(k)}(z)=\frac{\partial^{k}}{\partial \bar{\omega}^{k}} k(z, \omega)=\frac{z^{k}}{(1-z \bar{\omega})^{k+1}} .
$$

Then, since

$$
\left\langle f, \varphi_{\omega}^{(k)}\right\rangle_{\mathbf{H}_{2}}=f^{(k)}(\omega)
$$

it is readily checked that the set of all functions $h \in \mathbf{H}_{\mathbf{2}}$ for which property (ii) holds can be written as

$$
h(z)=h_{\min }(z)+\left(\frac{\dot{z}-w}{1-z \bar{\omega}}\right)^{N+1} u(z)
$$

D. Alpay: Ben Gurion Univ. of the Negev, Dep. Math., POB 653, Beer Sheva 84105 , Israel V. Bolotnikov: Ben Gurion Univ. of the Negev, Dep. Math., POB 653, Beer Sheva 84105, Israel 
with

$$
h_{\min }(z)=\left(\varphi^{(0)}(z), \varphi^{(1)}(z), \ldots, \varphi^{(N)}(z)\right) P^{-1}\left(\begin{array}{c}
h_{0} \\
\vdots \\
h_{N}
\end{array}\right)
$$

$P$ being the matrix with $i j$-entry

$$
P_{i j}=\left\langle{ }^{(i)}, \varphi_{\omega}^{(j)}\right\rangle_{\mathbf{H}_{2}}=\left.\frac{\partial^{i+j}}{\partial z^{j} \partial \bar{\omega}^{i}} k(z, \omega)\right|_{x=\omega}
$$

and $\mathbf{u}$ being a free parameter in $\mathbf{H}_{2}$.

Furthermore,

$$
\|h\|_{\mathbf{H}_{2}}^{2}=\left\|h_{\min }\right\|_{\mathbf{H}_{2}}^{2}+\|u\|_{\mathbf{H}_{2}}^{2}
$$

with

$$
\left\|h_{\min }\right\|_{\mathbf{H}_{2}}^{2}=\left(\bar{h}_{0}, \ldots, \bar{h}_{N}\right) P^{-1}\left(\begin{array}{c}
h_{0} \\
\vdots \\
h_{N}
\end{array}\right)
$$

This allows to take care of constraint (i). There is a solution $h$ of the CarathéodoryFéjer problem with $\|h\|_{\mathbf{H}_{2}} \leq 1$ if and only if $\left\|h_{\min }\right\|_{\mathbf{H}_{2}} \leq 1$. Then, all solutions of the problem are given by

$$
h(z)=h_{\min }(z)+\left(\frac{z-\omega}{1-z \bar{\omega}}\right)^{N}\left(1-\left\|h_{\min }\right\|_{\mathbf{H}_{2}}^{2}\right)^{1 / 2} u(z)
$$

with $\|u\|_{\mathbf{H}_{2}} \leq 1$.

The purpose of this paper is to study a matrix-valued version of this problem. We denote by $\boldsymbol{C}^{p \times q}$ the space of $p$-rows, $q$-columns matrices with complex entries, by $I_{q}$ the identity of $\boldsymbol{C}^{q \times q}$ and write $\boldsymbol{C}^{p}$ for $\boldsymbol{C}^{p \times 1}$. We set $\mathbf{H}_{2}^{p \times q}$ to be the space of $\boldsymbol{C}^{p \times q}$-valued functions with entries in $\mathbf{H}_{2}$. On the space $\mathbf{H}_{2}^{p \times q}$ we define the inner product

$$
\langle F, G\rangle=\frac{1}{2 \pi} \int_{0}^{2 \pi} \operatorname{Tr}\left\{G\left(e^{i t}\right)^{*} F\left(e^{i t}\right)\right\} d t
$$

and the matrix-valued Hermitian form

$$
[F, G]=\frac{1}{2 \pi} \int_{0}^{2 \pi} G\left(e^{i t}\right) F\left(e^{i t}\right) d t
$$

When $p=q=1$, then $\langle F, G\rangle=[F, G]$, but the conditions $\langle F, F\rangle \leq 1$ and $[F, F] \leq I_{q}$ are in general not equivalent for $q>1$ or $p>1$.

$\dot{A}$ matrix valued version of the Carathéodory-Féjer problem for $\mathrm{H}_{2}$ functions is the following 
Problem 1.1: Given matrices $c_{0}, \ldots, c_{n} \in C^{r \times p}$ and $d_{0}, \ldots, d_{n} \in C^{r \times q}$ and given $\omega \in \mathbb{D}$ describe the set of all functions $H \in \mathrm{H}_{2}^{p \times q}$ such that

$$
[H, H] \leq I_{q} \quad \text { and } \quad \sum_{i=0}^{k} c_{i} \frac{H^{(k-i)}(\omega)}{k !}=d_{k} \quad(k=0, \ldots, n) .
$$

To solve this problem we use a characterization of elements $H \in \mathbf{H}_{2}^{p \times q}$ for which $[H, H] \leq I_{q}$ in terms of Schur functions and apply the Fundamental Matrix Inequality method (FMI) developed by I. Kovalishina and V. Potapov (see [11 - 13]).

We will solve a problem more general than Problem 1.1, set in a framework developed in the papers [3 - 5] and which, in particular, allows to treat in a unified way the case of Hardy spaces of the unit disk and of a half plane.

The outline of the paper is as follows.

The paper consists of four sections. This introduction is the first one. In Section 2, we review the above mentioned framework and prove a number of results of [2] in this context. The general problem to be solved is then described. In Section 3 we give a necessary and sufficient condition for the problem to be solvable, in terms of a matrix inequality. In Section 4 we give an explicit description in terms of a linear fractional transformation.

\section{The spaces $H_{p}^{p \times q}\left(I_{q}\right)$}

Let $a, b$ be a pair of functions which are analytic in an open subset $\Omega \subset C$. Let

$$
\rho_{\omega}(\lambda)=a(\lambda) a(\omega)^{*}-b(\lambda) b(\omega)^{*}
$$

and let the subsets

$$
\Omega_{+}=\left\{\lambda \in \Omega: \rho_{\lambda}(\lambda)>0\right\} \quad \text { and } \quad \Omega_{-}=\left\{\lambda \in \Omega: \rho_{\lambda}(\lambda)<0\right\}
$$

be non-empty. Then there exists a point $\mu$ such that $|a(\mu)|=|b(\mu)| \neq 0$ and hence, the subset

$$
\Omega_{0}=\left\{\lambda \in \Omega: \rho_{\lambda}(\lambda)=0\right\}
$$

is non-empty.

The kernel $\rho_{\omega}(\lambda)^{-1}$ is non-negative in $\Omega_{+}$: for every choice of integer $k \in \mathbb{N}$ and of points $\omega_{1}, \ldots, \omega_{k}$ in $\Omega_{+}$, the $k \times k$ Hermitian matrix with $i j$-entry $\rho_{\omega_{i}}\left(\omega_{j}\right)^{-1}$ is non-negative. Therefore, there exists a reproducing kernel Hilbert space $\mathbf{H}_{\rho}$ with the reproducing kernel $\rho_{\omega}(\lambda)^{-1}$. The following direct characterization of the space $\mathbf{H}_{\rho}$ is given in [4: pp. $127-128]$.

Theorem 2.1: The space $\mathbf{H}_{\rho}$ consists of functions $f$ which are analytic on $\Omega_{+}$and admit a representation of the form

$$
f(\lambda)=\frac{1}{a(\lambda)} \sum_{n=0}^{\infty} c_{n} \sigma(\lambda)^{n}
$$


with

$$
\|f\|_{\mathbf{H},}^{2}=\sum_{n=0}^{\infty}\left|c_{n}\right|^{2}<\infty
$$

and where

$$
\sigma(\lambda)=\frac{b(\lambda)}{a(\lambda)}
$$

We refer to the papers [3 - 5] for further properties of the spaces $\mathbf{H}_{\rho}$ and for various examples of $\rho$. Here we mention that the representation (2.1) is essentially unique, i.e. if $\rho_{\omega}(\lambda)=c(\lambda) c(\omega)^{*}-d(\lambda) d(\omega)^{*}$ is another representation of $\rho$, then $(a(\lambda), b(\lambda))=$ $(c(\lambda), d(\lambda)) M$ where $M$ is a $\left(\begin{array}{cc}1 & 0 \\ 0 & -1\end{array}\right)$-unitary matrix.

Besides the case $a(\lambda)=1$ and $b(\lambda)=\lambda$ (for which $\Omega_{+}=D$ and $\Omega_{0}$ is the unit circle $\boldsymbol{T})$ the following two cases are of interest:

$$
a(\lambda)=1-i \lambda \quad \text { and } \quad b(\lambda)=1+i \lambda
$$

and

$$
a(\lambda)=\lambda+i\left(\lambda^{2}+1\right) \quad \text { and } \quad b(\lambda)=\lambda-i\left(\lambda^{2}+1\right) .
$$

In the first case, $\rho_{\omega}(\lambda)=-i(\lambda-\bar{\omega})$. Then, $\Omega_{+}=\boldsymbol{C}_{+}$(the open upper half plane) and $\Omega_{0}=\mathbb{R}$. In the second case, $\rho_{\omega}(\lambda)=-i(\lambda-\bar{\omega})(1-\lambda \bar{\omega}), \Omega_{0}=\mathbb{R} \cup \boldsymbol{T}$ and $\Omega_{+}=\left(\mathbb{D} \cap \boldsymbol{C}_{+}\right) \cup\left(\mathbb{E} \cap \boldsymbol{C}_{-}\right)$(where we have denoted by $\mathbb{E}$ the complementary of the closed unit disk and by $\boldsymbol{C}_{-}$the open lower half plane). Note that, in this case, $\Omega_{+}$is not connected.

We denote by $\mathbf{H}_{\rho}^{p \times q}$ the space of $p \times q$ matrices with entries in $\mathbf{H}_{\rho}$. Thus, an element in $\mathbf{H}_{\rho}^{p \times q}$ can be written as

$$
H(\lambda)=\frac{1}{a(\lambda)} \sum_{n=0}^{\infty} H_{n} \sigma(\lambda)^{n}
$$

with $H_{n} \in \boldsymbol{C}^{p \times q}$. On $\mathbf{H}_{p}^{p \times q}$ we define

$$
\langle H, G\rangle_{\mathbf{H}_{p}^{p \times q}}=\operatorname{Tr}\left(\sum_{n=0}^{\infty} G_{n}^{*} H_{n}\right)
$$

and

$$
[H, G]_{\mathbf{H}_{p}^{p \times q}}=\sum_{n=0}^{\infty} G_{n}^{*} H_{n} .
$$

The representation (2.6) of $H$ depends on the given representation (2.1) of $\rho$, but (2.7) and (2.8) are independent of the choice of $a$ and $b$ defining $\rho$. Indeed, we first consider the case $q=1$ and denote $\mathbf{H}_{\rho}^{p \times 1}$ by $\mathbf{H}_{\rho}^{p}$. The space $\mathbf{H}_{\rho}^{p}$ is the reproducing kernel Hilbert space of $\boldsymbol{C}^{p}$-valued functions with reproducing kernel $I_{p} / \rho_{\omega}(\lambda)$. Since there is only one such Hilbert space, definition (2.7) is independent of the choice of $a$ and $b$ when $q=1$. To study the case $q>1$, take $H \in \mathbf{H}_{\rho}^{p \times q}$ of the form (2.6) and $c \in \boldsymbol{C}^{q}$. Then $H c \in \mathbf{H}_{\rho}^{p}$ and so, for every $c, \sum_{n=0}^{\infty} c^{*} H_{n}^{*} H_{n} c$ is independent of the given realization of $\rho$. Hence 
(2.8), and therefore (2.7), are independent of the given realizations of $\rho$. We denote by $\mathbf{H}_{\rho}^{p \times q}\left(I_{q}\right)$ the space of functions $H \in \mathbf{H}_{\rho}^{p \times q}$ for which $[H, H]_{\mathbf{H}_{p}^{p \times q}} \leq I_{q}$ and now give two characterizations of its elements. We first define $S_{\rho}^{p \times q}$ to be the set of all $\boldsymbol{C}^{p \times q}$-valued functions $S$ for which the kernel

$$
\frac{I_{p}-S(\lambda) S(\omega)^{*}}{\rho_{\omega}(\lambda)}
$$

is non-negative on $\Omega_{+}$. Equivalently, the operator of multiplication by $S$ is a contraction from $\mathbf{H}_{\rho}^{q}$ into $\mathbf{H}_{\rho}^{p}$ (see [4: p. 128, Theorem 3.1]).

Theorem 2.2: Let $H$ be a $C^{p \times q}$-valued function defined in $\Omega_{+}$. Then $H$ belongs to the space $\mathbf{H}_{p}^{p \times q}\left(I_{q}\right)$ (and is in particular analytic in $\Omega_{+}$) if and only if the kernel

$$
K_{H}(\lambda, \omega)=\frac{I_{p}}{\rho_{\omega}(\lambda)}-H(\lambda) H(\omega)^{*}
$$

is non-negative in $\Omega_{+}$.

The Proof follows from that given in [2] for $\rho_{\omega}(\lambda)=1-\lambda \bar{\omega}$ and relies on the fact that $[H, H]_{\mathbf{H}_{\rho}^{p \times q}} \leq I_{q}$ if and only if the multiplication operator $c \longrightarrow H c$ is a contraction from $\boldsymbol{C}^{q}$ into $\mathbf{H}_{\rho}^{p}$. Let us remark that in the scalar case $p=q=1$ the result above has been proved by D. Sarason [14 - 16].

Theorem 2.3: Let $H$ be a $\boldsymbol{C}^{p \times q}$-valued function analytic in $\Omega_{+}$. It:belongs to the space $\mathbf{H}_{\rho}^{p \times q}\left(I_{q}\right)$ if and only if it can be written as

$$
H(\lambda)=S_{1}(\lambda)\left(a(\lambda) I_{q}-b(\lambda) S_{2}(\lambda)\right)^{-1}
$$

where

$$
S=\left(\begin{array}{c}
S_{1} \\
S_{2}
\end{array}\right) \in \mathbf{S}_{p}^{(p+q) \times q} .
$$

The proof which will be given below follows the case $a(\lambda)=1$ and $b(\lambda)=\lambda$ which was considered in [2]. We need for the proof the following lemma.

Lemma 2.4: Let $U$ be an open subset of $\mathbb{D}$ and let $H$ be a $C^{p \times q}$-valued function defined on $U$ and such that the function

$$
\tilde{K}_{H}(z, \nu)=\frac{I_{p}}{1-z \bar{\nu}}-H(z) H(\nu)^{*}
$$

is non-negative in $U$. Then $H$ is the restriction of a (unique) function $\hat{H} \in \mathbf{H}_{2}^{p \times q}\left(I_{q}\right.$ ). In particular, $H$ is analytic in $U$.

Proof: Let $k_{\nu}(z)=1 /(1-z \bar{\nu})$ and let us introduce a densely defined relation $T$ on the space $\mathbf{H}_{2}^{p}$ by

$$
T\left(k_{\nu} c\right)=H(\nu)^{*} c \quad\left(\nu \in U, c \in \boldsymbol{C}^{p}\right)
$$


Since $\tilde{K}_{H}(z, \nu)$ is positive, $T$ is in fact a contraction operator and therefore extends to a contraction from $\boldsymbol{C}^{p}$ into $\mathbf{H}_{2}^{p}$. For $\nu \in U$ and $c, d \in \boldsymbol{C}^{p}$ we have

$$
c^{*}\left(T^{*} d\right)(\nu)=\left\langle T^{*} d, k_{\nu}\right\rangle_{\mathbf{H}_{2}^{p}}=\left\langle d, H(\nu)^{*} c\right\rangle_{\boldsymbol{C}^{p}}=c^{*} H(\nu) d
$$

and thus $H$ is the restriction to $U$ of the function $\hat{H} \in \mathbf{H}_{2}^{p \times q}$ defined by $\hat{H}(v) d=$ $\left(T^{*} d\right)(v)$. To conclude, it remains to check that the kernel $\tilde{K}_{\dot{H}}(z, \omega)$ is non-negative in $\mathbb{D}$, but this follows from the fact that $T^{*}$ is a contraction and from the formula $c_{1}^{*} K_{\dot{H}}(z, \nu) c_{2}=\left(I-T^{*} T\right)\left\langle k_{\nu} c_{1}, k_{z} c_{2}\right\rangle_{\mathbf{H}_{2}^{p}}$ for elements $c_{1}, c_{2} \in \boldsymbol{C}^{p}$

Proof of Theorem 2.3: Let us first assume that $H$ is of the form (2.11). Then,

$$
K_{H}(\lambda, \omega)=A(\dot{\lambda})\left\{\frac{I_{p+q}-S(\lambda) S(\omega)^{*}}{\rho_{\omega}(\lambda)}\right\} A(\omega)^{*}
$$

with $A(\lambda)=\left(I_{p}, \lambda H(\lambda)\right)$ and therefore, $K_{H}(\lambda, \omega)$ is non-negative in $\Omega_{+}$. To study the converse, we note that by (2.6) the function $H$ can be written as $H(\lambda)=a(\lambda)^{-1} \phi(\sigma(\lambda))$ where $\phi \in \mathbf{H}_{2}^{p \times q}$. From the non-negativity of the kernel $K_{H}(\lambda, \omega)$ it follows that the function

$$
\frac{1}{1-\sigma(\lambda) \sigma(\omega)^{*}}-\phi(\sigma(\lambda)) \phi(\sigma(\omega))^{*}
$$

is non-negative in $\Omega_{+}$. Thus, the function $(1-z \bar{\nu})^{-1}-\phi(z) \phi(\bar{\nu})^{*}$ is non-negative in a subset of $I D$ and, by Lemma 2.1 , in all of $D$. We apply the result of [2] to obtain that $\phi$ admits a representation $\phi(z)=s_{1}(z)\left(I-z s_{2}(z)\right)^{-1}$ where $s=\left(\begin{array}{l}s_{1} \\ s_{2}\end{array}\right) \in \mathbf{S}^{(p+q) \times q}$. We now set $S(\lambda)=\cdot s(\sigma(\lambda))$. Then, $S \in \mathbf{S}_{\rho}^{(p+q) \times q}$ and (2.11) holds

To conclude this section we define the following interpolation problem.

Problem 2.5: Given matrices $c_{i} \in \boldsymbol{C}^{r \times p}$ and $d_{i} \in \boldsymbol{C}^{r \times q}(i=0, \ldots, n)$ and given point $\lambda_{0} \in \Omega_{+}$such that

$$
\delta=a^{\prime}\left(\lambda_{0}\right) b\left(\lambda_{0}\right)-b^{\prime}\left(\lambda_{0}\right) a\left(\lambda_{0}\right) \neq 0
$$

find necessary and sufficient condition to insure the existence of a function

$$
H(\lambda)=\sum_{i=0}^{\infty} H_{i}\left(\lambda-\lambda_{0}\right)^{i}
$$

in the space $\mathbf{H}_{\rho}^{p \times q}\left(I_{q}\right)$ such that

$$
\sum_{i=0}^{k} c_{i} H_{k-i}=d_{k} \quad(k=0, \ldots, n)
$$

and describe the set of all such functions when this condition is met.

Using Theorem 2.3 we reduce the initial problem $I\left(\mathbf{H}_{p}^{p \times q}\left(I_{q}\right)\right)$ to an interpolation problem $I\left(\mathbf{S}^{(p+q) \times q}\right)$ in the Schur class $\mathbf{S}_{\rho}^{(p+q) \times q}$. 
To every $\boldsymbol{C}^{r \times m}$-valued function $V(\lambda)=\sum_{i=0}^{\infty} V_{i}\left(\lambda-\lambda_{0}\right)^{i}$ analytic at $\lambda_{0}$ we associate the lower triangular Toeplitz block matrices

$$
L_{k}(V)=\left(\begin{array}{cccc}
V_{0} & 0 & \ldots & 0 \\
V_{1} & \ddots & \ddots & \vdots \\
\vdots & \ddots & \ddots & 0 \\
V_{k} & \ldots & V_{1} & V_{0}
\end{array}\right) \quad(k=0,1, \ldots)
$$

The following auxiliary results can be checked by a direct computation.

Lemma 2.6: Let $V$ and $U$ be matrix-valued functions analytic at $\lambda_{0}$ and let $L_{k}(V)$ and $L_{k}(U)$ be associated matrices defined by (2.15). Then

(i) $L_{k}(U V)=L_{k}(U) L_{k}(V)$

(ii) $L_{k}(U+V)=L_{k}(U)+L_{k}(V)$

(iii) $L_{k}(U)=L_{k}(V)$ if and only if $U_{i}=V_{i}(i=0, \ldots, k)$.

(iv). $\operatorname{det} L_{k}(V) \neq 0$ if and only if $\operatorname{det} V_{0} \doteq \operatorname{det} V\left(\lambda_{0}\right) \neq 0$.

Lemma 2.7: Let. $H$ and $S$ be the functions defined by (2.11) which belong to the spaces $\mathbf{H}_{p}^{p \times q}\left(I_{q}\right)$ and $\mathbf{S}_{\rho}^{(p+q) \times q}$, respectively. Then $H$ satisfies condition (2.14) if and only if $S$ satisfies the interpolation conditions

$$
\sum_{i=0}^{k}\left(c_{i}, b_{0} d_{i}+b_{1} d_{i-1}+\ldots+b_{i} d_{0}\right) S_{k-i}=a_{0} d_{k}+a_{1} d_{k-1}+\ldots+a_{k} d_{0}
$$

where $a_{i}$ and $b_{i}$ are coefficients from the Taylor expansions

$$
a(\lambda)=\sum_{i=0}^{\infty} a_{i}\left(\lambda-\lambda_{0}\right)^{i} \quad \text { and } \quad b(\lambda)=\sum_{i=0}^{\infty} b_{i}\left(\lambda-\lambda_{0}\right)^{i}
$$

of $a$ and $b$, respectively, in a neighbourhood of $\lambda_{0}$.

Proof: Let us introduce the matrix polynomials

$$
C(\lambda)=\sum_{i=0}^{n} c_{i}\left(\lambda-\lambda_{0}\right)^{i} \quad \text { and } \quad D(\lambda)=\sum_{i=0}^{n} d_{i}\left(\lambda-\lambda_{0}\right)^{i}
$$

built from the interpolation data $c_{i}$ and $d_{i}$, respectively. Using triangular matrices $L_{k}$ from (2.15) one can rewrite the interpolation conditions (2.14) and (2.16) as

$$
L_{n}(C H)=L_{n}(D)
$$

and

$$
L_{n}((C, b D) S)=L_{n}(a D)
$$


respectively. It follows from (2.11) by Lemma 2.7 that

$$
L_{n}(H) L_{n}\left(a I_{g}-b S_{2}\right)=L_{n}\left(S_{1}\right)
$$

Let (2.19) hold. Multiplying (2.21) by the matrix $L_{n}(C)$ on the left and using (2.19) we obtain $L_{n}(D) L_{n}\left(a I_{q}-b S_{2}\right)=L_{n}\left(C S_{1}\right)$ which in view of Lemma 2.7 can be rewritten as

$$
L_{n}\left(C S_{1}+b D S_{2}\right)=L_{n}(a D)
$$

and is equivalent to (2.20) due to the decomposition (2.11) of $S$.

Let, conversely, (2.20) (or, equivalently, (2.22)) hold. Multiplying (2.21) by $L_{n}(C)$ on the left and comparing the obtained equality with (2.22) we get

$$
L_{n}(C H) L_{n}\left(a I_{q}-b S_{2}\right)=L_{n}(D) L_{n}\left(a I_{q}-b S_{2}\right)
$$

which in view of the non-degeneracy of the matrix $L_{n}\left(a I_{q}-b S_{2}\right)$ is equivalent to (2.19).

We thus have to solve an interpolation problem $I\left(\mathbf{S}_{\rho}^{p \times q}\right)$ in the $\operatorname{Schur}$ class $\mathbf{S}_{\rho}^{p \times q}$ and with the interpolation conditions (2.16). A special version of this problem was solved in [3] using the reproducing kernel method, and the arguments of [3] can be easily adapted for the present situation. For completeness, we present the independent solution based on the Potapov method of the fundamental matrix inequality (see [8: pp. 252 - 267] and $[11-13])$.

\section{The fundamental matrix inequality}

We begin with the analogue of the Schwartz-Pick inequality for functions of the class $\mathbf{S}_{\rho}^{(p+q) \times q}$ (which is well known for the classical case $\rho_{\omega}(\lambda)=1-\lambda \bar{\omega}$ and was proved in the paper [1]).

Lemma 3.1: Let $S \in \mathrm{S}_{\rho}^{(p+q) \times q}$ and let for points $\omega_{0}, \ldots, \omega_{n} \in \Omega_{+}$

$$
b\left(\omega_{i}\right) a\left(\omega_{j}\right) \neq a\left(\omega_{i}\right) b\left(\omega_{j}\right) \quad(i \neq j) .
$$

Then the kernel

$$
\mathbf{T}_{S}(\lambda, \omega)=\left(\begin{array}{cc}
T & \hat{G}(\lambda) \hat{M} J\left(\begin{array}{c}
S(\lambda) \\
I_{q}
\end{array}\right) \\
\left(S(\omega)^{*}, I_{q}\right) J \hat{M}^{*} \hat{G}(\omega)^{*} & \frac{I_{q}-S(\omega)^{*} S(\lambda)}{\rho_{\omega}(\lambda)}
\end{array}\right)
$$

is non-negative on $\Omega_{+}$, where

$$
\begin{gathered}
\hat{M}=\left(\begin{array}{cc}
I_{p+q} & S\left(\omega_{0}\right) \\
\vdots & \vdots \\
I_{p+q} & S\left(\omega_{n}\right)
\end{array}\right) \quad \text { and } \quad J=\left(\begin{array}{cc}
I_{p+q} & 0 \\
0 & -I_{q}
\end{array}\right) \\
\hat{G}(\lambda)=(b(\lambda) \hat{A}-a(\lambda) \hat{B})^{-1}
\end{gathered}
$$




$$
\hat{A}=\operatorname{diag}\left(a\left(\omega_{i}\right) I_{p+q}\right)_{i=0}^{n} \quad \text { and } \quad \hat{B}=\operatorname{diag}\left(b\left(\omega_{i}\right) I_{p+q}\right)_{i=0}^{n}
$$

and $T$ is the block matrix defined by

$$
T_{i j}=\frac{I_{p+q}-S\left(\omega_{i}\right) S\left(\omega_{j}\right)^{*}}{\rho_{\omega_{j}}\left(\omega_{i}\right)} \quad(i, j=1, \ldots, n) .
$$

Remark 3.2: The matrices $\hat{M}, J, \hat{A}, \hat{B}$ and $T$ defined by (3.3), (3.5) and (3.6) satisfy the identity

$$
\hat{A} T \hat{A}^{*}-\hat{B} T \hat{B}^{*}=\hat{M} J \hat{M}^{*}
$$

which can be checked by a direct computation.

To describe the set of all solutions to the problem $I\left(\mathbf{S}_{\rho}^{(p+q) \times q}\right)$ in terms of a matrix inequality, we recall an auxiliary lemma from [7].

Lemma 3.3: Let $\omega_{0}, \ldots, \omega_{n}$ be different points in $C$ and let $\Phi_{\omega, p}$ be the lower triangular matrix with block entries

$$
\left(\Phi_{\omega, p}\right)_{i j}= \begin{cases}\varphi_{i}^{\prime}\left(\omega_{j}\right)^{-1} I_{p} & \text { for } i \geq j \\ 0 & \text { for } i<j\end{cases}
$$

where

$$
\varphi_{j}(\lambda)=\prod_{i=0}^{j}\left(\lambda-\omega_{i}\right) \quad(j=0, \ldots, n) .
$$

Let $u(\lambda)$ be a $C^{p \times q}$-valued analytic function with the expansion $u(\lambda)=\sum_{i=0}^{n} u_{i}\left(\lambda-\lambda_{0}\right)^{i}$ at $\lambda_{0} \in \boldsymbol{C}$. Then

$$
\lim _{\substack{\omega_{i} \rightarrow \lambda_{0} \\
i=\lambda_{1}, \ldots, n, p}} \Phi_{\omega, p}\left(\begin{array}{c}
u\left(\omega_{0}\right) \\
\vdots \\
u\left(\omega_{n}\right)
\end{array}\right)=\left(\begin{array}{c}
u_{0} \\
\vdots \\
u_{n}
\end{array}\right)
$$

and, moreover,

$$
\lim _{\substack{\omega_{i} \rightarrow \lambda_{0} \\
i=0,1, \ldots, n}} \Phi_{\omega, p}\left(\begin{array}{ccc}
u\left(\omega_{0}\right) & & 0 \\
\vdots & \ddots & \\
0 & & u\left(\omega_{n}\right)
\end{array}\right) F=\left(\begin{array}{cccc}
u_{0} & 0 & \cdots & 0 \\
u_{1} & \ddots & \ddots & \vdots \\
\vdots & \ddots & \ddots & 0 \\
u_{n} & \ldots & u_{1} & u_{0}
\end{array}\right) \lim _{\substack{\omega_{i} \rightarrow \lambda_{0} \\
i=0,1, \ldots, n}} \Phi_{\omega, q} F
$$

for every choice of the matrix $F$.

Theorem 3.4: Let $S=\sum_{i=0}^{\infty} S_{i}\left(\lambda-\lambda_{0}\right)^{i}$ be a $\boldsymbol{C}^{(p+q) \times q}$-valued function, let $\lambda_{0} \in$ $\Omega_{+}$be a point satisfying condition (2.12) and let $\rho_{\omega}(\lambda)$ be of the form (2.1) with a and $b$ given by (2.17). Then $S$ is a solution to the problem $I\left(\mathbf{S}_{\rho}^{(p+q) \times q}\right)$ if and only if the kernel

$$
\mathbf{K}_{S}(\lambda, \omega)=\left(\begin{array}{cc}
K & G(\lambda) M J\left(\begin{array}{c}
S(\lambda) \\
I_{q}
\end{array}\right) \\
\left(S(\omega)^{*} ; I_{q}\right) J M^{*} G(\omega)^{*} & \frac{I_{q}-S(\omega)^{*} S(\lambda)}{\rho_{\omega}(\lambda)}
\end{array}\right)
$$


is non-negative on $\Omega_{+}$, where

$$
\begin{gathered}
G(\lambda)=(b(\lambda) A-a(\lambda) B)^{-1} \\
A=\left(\begin{array}{ccc}
a_{0} I_{r} & & 0 \\
\vdots & \ddots & \\
a_{n} I_{r} & \ldots & a_{0} I_{r}
\end{array}\right) \quad \text { and } \quad B=\left(\begin{array}{ccc}
b_{0} I_{r} & & 0 \\
\vdots & \ddots & \\
b_{n} I_{r} & \ldots & b_{0} I_{r}
\end{array}\right) \\
M=(\mathbf{c}, B \mathbf{d}, A \mathbf{d})
\end{gathered}
$$

with

$$
\mathbf{c}=\left(\begin{array}{c}
c_{0} \\
\vdots \\
c_{n}
\end{array}\right) \quad \text { and } \quad \mathbf{d}=\left(\begin{array}{c}
d_{0} \\
\vdots \\
d_{n}
\end{array}\right)
$$

and

$$
K=\int_{\gamma}(z A-B)^{-1} M J M^{*}\left(z A^{*}-B^{*}\right)^{-1} d z
$$

where $\gamma$ is a simple closed contour in the unit disk around the point $b\left(\lambda_{0}\right) / a\left(\lambda_{0}\right)$.

Proof: Let $S$ be a solution to problem $I\left(\mathbf{S}_{\rho}^{(p+q) \times q}\right)$ and let $\omega_{0}, \ldots, \omega_{n} \in \Omega_{+}$be points from the neighborhood of $\lambda_{0}$ satisfying condition (3.1). By Lemma 3.1 the kernel $\mathbf{T}_{S}(\lambda, \omega)$ defined by (3.2) is non-negative on $\Omega_{+}$. Let $\hat{M}$ and $\Phi_{\omega, p}$ be matrices given by (3.3) and (3.8) and let

$$
C=\left(\begin{array}{ccc}
c_{0} & & 0 \\
\vdots & \ddots & \\
c_{n} & \cdots & c_{0}
\end{array}\right)
$$

Setting in formula (3.9) $u(\lambda)=\left(I_{p+q}, S(\lambda)\right)$ and taking into account all the furmulas (3.3), (3.13) - (3.15), (2.14) and (2.18), we obtain

$$
\lim _{\substack{\omega_{i} \rightarrow \lambda_{0} \\ i=0,1, \ldots, n}} C \Phi_{\omega, p} \hat{M}=M
$$

Furthermore, setting in (3.10) $u(\lambda)=a(\lambda) I_{p}$ and $u(\lambda)=b(\lambda) I_{p}$ and using formuals $(3.5),(3.13)$ and $(3.17)$ we receive respectively

$$
\lim _{\substack{i \rightarrow \lambda_{0} \\ i=0,1, \ldots, n}} C \Phi_{\omega, p} \hat{A} F=A C \lim _{\substack{\omega_{i} \rightarrow \lambda_{0} \\ i=0,1, \ldots, n}} \Phi_{\omega, p} F
$$

and

$$
\lim _{\substack{\omega_{i} \rightarrow \lambda_{0} \\ i=0 ; i, \ldots, n}} C \Phi_{\omega, p} \hat{B} F=B C \lim _{\substack{\omega_{i} \rightarrow \lambda_{0} \\ i=0_{i}, \ldots, n}} \Phi_{\omega, p} F
$$

for arbitrary matrix $F$. Setting in the two last equalities $F=\hat{M}$ and using (3.18) we come to the relations

$$
\lim _{\substack{\omega_{i} \rightarrow \lambda_{0} \\ i=0,1, \ldots, n}} C \Phi_{\omega, p} \hat{A} \hat{M}=A M \quad \text { and } \quad \lim _{\substack{\omega_{i} \rightarrow \lambda_{0} \\ i=0,1, \ldots, n}} C \Phi_{\omega, p} \hat{B} \hat{M}=B M
$$


Note that, in view of (3.4), (3.12) and (3.21),

$$
\lim _{\substack{\omega_{i} \rightarrow \lambda_{0} \\ i=0,1, \ldots, n}} C \Phi_{\omega, p} \hat{G}(\lambda) \hat{M}=G(\lambda) M
$$

Multiplying the non-negative kernel $\mathbf{T}_{S}(\lambda, \omega)$ from (3.2) by the matrix $\left(\begin{array}{cc}C \Phi_{\omega, p} & 0 \\ 0 & I_{p}\end{array}\right)$ on the left and by its adjoint on the right we set $\omega_{i} \rightarrow \lambda_{0}(i=0, \ldots, n)$. In view of (3.18) we obtain the non-negativity of the kernel $K_{S}(\lambda, \omega)$ defined by (3.11) with the block $K$ given by

$$
K=\lim _{\substack{\omega \rightarrow \lambda_{0} \\ i=0,1, \ldots, n}} C \Phi_{\omega, p} T \Phi_{\omega, p}^{*} C^{*} .
$$

To finish the necessity of theorem we have to show that matrices defined by (3.16) and (3.23) coincide. Indeed, multiplying the identity (3.7) by $C \Phi_{\omega, p}$ on the left, by $\Phi_{\omega, p}^{*} C^{*}$ on the right and setting $\omega_{i} \rightarrow \lambda_{0} \quad(i=0, \ldots, n)$ we obtain in view of $(3.18)-(3.20)$

$$
A K A^{*}-B K B^{*}=M J M^{*} \text {. }
$$

Since $\left|b_{0}\right|<\left|a_{0}\right|$, the matrix $K$ given by (3.23) is the unique solution of the Stein equation (3.24) and (see [6: Appendix]) admits a representation (3.16).

To prove the sufficiency of theorem we recall the following two lemmas.

Lemma 3.5 (see [7]): Let $a$ and $b$ be analytic functions with expansions (2.17) at $\lambda_{0}$, let $A$ and $B$ be associated Toeplitz matrices given by (3.13) and let the matrix $\Lambda \in C^{(n+1) r \times(n+1) r}$ be defined by

$$
\Lambda=\left(\begin{array}{cccc}
\lambda_{0} I_{r} & 0 & \ldots & 0 \\
I_{r} & \ddots & \ddots & \vdots \\
\vdots & \ddots & \ddots & 0 \\
0 & \ldots & I_{r} & \lambda_{0} I_{r}
\end{array}\right)
$$

Then

$$
b(\lambda) A-a(\lambda) B=(\lambda I-\Lambda) \Omega(\lambda)
$$

where

$$
\Omega(\lambda)=A \hat{B}(\lambda)-B \hat{A}(\lambda)
$$

and $\hat{A}(\lambda)$ and $\hat{B}(\lambda)$ are matrix-valued functions given by

$$
\begin{aligned}
& \hat{A}(\lambda)=\left(\begin{array}{ccc}
\hat{a}_{1}(\lambda) I_{r} & & 0 \\
\vdots & \ddots & \vdots \\
\hat{a}_{n+1}(\lambda) I_{r} & \ldots & \hat{a}(\lambda) I_{r}
\end{array}\right) \quad \text { with } \hat{a}_{j}(\lambda)=\sum_{i=0}^{\infty} a_{j+i}\left(\lambda-\lambda_{0}\right)^{i} \\
& \hat{B}(\lambda)=\left(\begin{array}{ccc}
\hat{b}_{1}(\lambda) I_{r} & & 0 \\
\vdots & \ddots & \\
\hat{b}_{n+1}(\lambda) I_{r} & \ldots & \hat{b}(\lambda) I_{r}
\end{array}\right) \quad \text { with } \hat{b}_{j}(\lambda)=\sum_{i=0}^{\infty} b_{j+i}\left(\lambda-\lambda_{0}\right)^{i} .
\end{aligned}
$$


Remark 3.6: It follows from (3.27) - (3.28) that $\Omega(\lambda)$ is a lower triangular Toeplitz block-matrix with diagonal elements equal to $a_{0} \hat{b}_{1}(\lambda)-b_{0} \hat{a}_{1}(\lambda)$. So, $\Omega(\lambda)$ is invertible if and only if $a_{0} \hat{b}_{1}(\lambda) \neq b_{0} \hat{a}_{1}(\lambda)$. In particular, the diagonal elements of the matrix $\Omega\left(\lambda_{0}\right)$ are equal to $\delta$ (see (2.12)) and hence,

$$
\operatorname{det} \Omega\left(\lambda_{0}\right) \neq 0 \text {. }
$$

Lemma 3.7 (see [7]): Let $u(\lambda)=\sum_{i=0}^{\infty} u_{i}\left(\lambda-\lambda_{0}\right)^{i}$ be a $\boldsymbol{C}^{p \times m} \cdot$ valued function, let $w_{0}, \ldots, w_{n}$ be arbitrary $r \times p$-matrices and let $\Lambda$ be the mairix defined by (3.25). Then, for $\lambda \rightarrow \lambda_{0}$,

$$
(\lambda I-\Lambda)^{-1}\left(\begin{array}{c}
w_{0} \\
\vdots \\
w_{n}
\end{array}\right) u(\lambda)=(\lambda I-\Lambda)^{-1}\left(\begin{array}{c}
w_{0} u_{0} \\
w_{0} u_{1}+w_{1} u_{0} \\
\vdots \\
w_{0} u_{n}+\ldots+w_{n} u_{0}
\end{array}\right)+O(1)
$$

If, moreover, the function

$$
(\lambda I-\Lambda)^{-1}\left(\begin{array}{c}
w_{1} \\
\vdots \\
w_{n}
\end{array}\right) u(\lambda)
$$

is analytic at $\lambda_{0}$, then

$$
\sum_{i=0}^{k} w_{i} u_{k-i}=0 \quad(k=0, \ldots, n) .
$$

Let now $S$ be an analytic $\boldsymbol{C}^{(p+q) \times q}$-valued function such that the kernel $\mathbf{K}_{S}(\lambda, \omega)$ defined by (3.11) is non-negative on $\Omega$. Then the kernel $\left(I_{q}-S(\bar{\lambda})^{*} S(\bar{\omega})\right) / \rho_{\omega}(\lambda)$ is also non-negative on $\Omega_{+}$and hence, $S \in \mathrm{S}_{\rho}^{(p+q) \times q}$. It remains to show that the function $S$ satisfies the interpolation conditions (2.16). Since the kernel $\mathbf{K}_{S}(\lambda, \omega)$ is non-negative, the function $G(\lambda) M J\left(\begin{array}{c}S(\lambda) \\ I_{q}\end{array}\right)$ is bounded (and therefore analytic) in compact neighbourhoods of $\lambda_{0}$. Using the factorization (3.26) and taking into account (3.29) we conclude that the function $(\lambda I-\Lambda)^{-1} M J\left(\begin{array}{c}S(\lambda) \\ I_{q}\end{array}\right)$ is analytic at $\lambda_{0}$. Applying Lemma 3.7 for

$$
u(\lambda)=\left(\begin{array}{c}
S(\lambda) \\
I_{q}
\end{array}\right) \quad \text { and } \quad w_{k}=\left(c_{k}, \sum_{i=0}^{k} b_{i} d_{k-i},-\sum_{i=0}^{k} a_{i} d_{k-i}\right)
$$

we obtain (see (3.32))

$$
\sum_{i=0}^{k}\left\{\left(c_{i}, \sum_{j=0}^{i} b_{j} d_{i-j}\right) S_{k-i}-a_{i} d_{k-i}\right\}=0 \quad(k=0, \ldots, n)
$$

which coincides with (2.16) 


\section{Description of all solutions}

In this section we parametrize the set of all solutions to the problem $I\left(\mathbf{H}_{\rho}^{p \times q}\left(I_{q}\right)\right)$ in terms of the linear fractional transformation under the hypothesis that its informative matrix $K$ is strictly positive. Let us consider the $C^{(p+2 q) \times(p+2 q)}$-valued function $\Theta$ defined by

$$
\Theta(\lambda)=I_{p+2 q}+\rho_{\mu}(\lambda) M^{*}\left(a(\lambda) A^{*}-b(\lambda) B^{*}\right)^{-1} K^{-1}\left(a(\mu)^{*} A-b(\mu)^{*} B\right)^{-1} M J
$$

where matrices $A, B, M$ are defined by (3.13) and (3.14) and where $\mu$ is an arbitrary point in $\Omega_{0}$ such that $|a(\mu)|=|b(\mu)| \neq 0$. Due to the identity (3.24) (see [1: pp. 223 224]) the function $\Theta$ is $J$-inner in $\Omega_{+}$and moreover,

$$
\Theta(\lambda)^{-*} J \Theta(\omega)-J=\rho_{\omega}(\lambda) J M^{*} G(\omega)^{*} K^{-1} G(\lambda) M J
$$

Since $K$ is invertible, the non-negativity of the kernel $K_{S}(\lambda, \omega)$ given by (3.11) is equivalent to the non-negativity of the kernel

$$
\tilde{\mathbf{K}}_{S}(\lambda, \omega)=\left(S(\bar{\lambda})^{*}, I_{q}\right)\left\{\frac{J}{\rho_{\omega}(\lambda)}-J M^{*} G(\bar{\lambda})^{*} K^{-1} G(\bar{\omega}) M J\right\}\left(\begin{array}{c}
S(\bar{\omega}) \\
I_{q}
\end{array}\right)
$$

which in view of (4.2) can be rewritten as

$$
\tilde{\mathbf{K}}_{S}(\lambda, \omega)=\left(S(\bar{\lambda})^{*}, I_{p}\right) \frac{\Theta(\bar{\lambda})^{-*} J \Theta^{-1}(\bar{\omega})}{\rho_{\omega}(\lambda)}\left(\begin{array}{c}
S(\lambda) \\
I_{q}
\end{array}\right)
$$

The set of all functions $S$ such that the corresponding kernel $\tilde{\mathbf{K}}_{S}(\lambda, \omega)$ is non-negative on $\Omega_{+}$(or, equivalently, the set of all solutions to the problem $I\left(\mathbf{S}_{\rho}^{(p+q) \times q}\right)$ ) was described in [3].

Theorem 4.1: Let

$$
\Theta=\left(\begin{array}{ll}
\theta_{11} & \theta_{12} \\
\theta_{21} & \theta_{22}
\end{array}\right):\left(\begin{array}{c}
\boldsymbol{C}^{p+q} \\
\boldsymbol{C}^{q}
\end{array}\right) \rightarrow\left(\begin{array}{c}
\boldsymbol{C}^{p+q} \\
\boldsymbol{C}^{q}
\end{array}\right)
$$

be the block decomposition of the function $\Theta$ given by (4.1). Then the linear fractional transformation

$$
S(\lambda)=\left(\theta_{11}(\lambda) \sigma(\lambda)+\theta_{12}(\lambda)\right)\left(\theta_{21}(\lambda) \sigma(\lambda)+\theta_{22}(\lambda)\right)^{-1}
$$

gives the parametrization of all solutions to the problem $I\left(\mathbf{S}_{\rho}^{(p+q) \times q}\right)$ when the parameter $\sigma$ varies in $\mathbf{S}_{\rho}^{(p+q) \times q}$.

Combining Theorem 2.3 and Theorem 4.1 we obtain a similar description for the solutions to the problem $I\left(\mathbf{H}_{\boldsymbol{p}}^{p \times q}\left(I_{q}\right)\right)$. 
Theorem 4.2: Let

$$
\Psi=\left(\begin{array}{ll}
\psi_{11} & \psi_{12} \\
\psi_{21} & \psi_{22}
\end{array}\right):\left(\begin{array}{c}
\boldsymbol{C}^{p+q} \\
\boldsymbol{C}^{q}
\end{array}\right) \rightarrow\left(\begin{array}{l}
\boldsymbol{C}^{p} \\
\boldsymbol{C}^{q}
\end{array}\right)
$$

be the block decomposition of the $\boldsymbol{C}^{(p+q) \times(p+2 q)}$-valued function

$$
\begin{aligned}
\Psi(\lambda)= & \left(\begin{array}{ccc}
I_{p} & 0 & 0 \\
0 & -b(\lambda) I_{q} & a(\lambda) I_{q}
\end{array}\right) \\
& +\rho_{\mu}(\lambda)\left(\begin{array}{c}
\mathbf{c}^{*}\left(a(\lambda) A^{*}-b(\lambda) B^{*}\right. \\
\mathrm{d}^{*}
\end{array}\right) K^{-1}\left(a(\mu)^{*} A-b(\mu)^{*} B\right)^{-1} M J
\end{aligned}
$$

where $A, B, M, \mathrm{c}$ and $\mathbf{d}$ are matrices given by (3.13)-(3.15). Then the linear fractional transformation

$$
H(\lambda)=\left(\psi_{11}(\lambda) \sigma(\lambda)+\psi_{12}(\lambda)\right)\left(\psi_{21}(\lambda) \sigma(\lambda)+\psi_{22}(\lambda)\right)^{-1}
$$

gives a parametrization of all solutions to the problem $I\left(\mathbf{H}_{p}^{p \times q}\left(I_{q}\right)\right)$ when the parameter $\sigma$ varies in $\mathrm{S}_{\rho}^{(p+q) \times q}$.

Proof: By Theorem 2.3 and Lemma 2.7, $H$ is a solution to the problem $I\left(\mathbf{H}_{\rho}^{p \times q}\left(I_{q}\right)\right)$ if and only if it admits a representation (2.10) for some solution $S$ to the problem $I\left(\mathbf{S}_{\rho}^{(p+q) \times q}\right)$. By Theorem 4.1,S solves problem $I\left(\mathbf{S}_{\rho}^{(p+q) \times q}\right)$ if and only if it is of the form (4.4) for some $\sigma \in S_{\rho}^{(p+q) \times q}$. It follows from (2.11) that

$$
S_{1}(z)=\left(I_{p}, O_{p \times q}\right) S(z) \quad \text { and } \quad S_{2}(z)=\left(O_{q \times p}, I_{q}\right) S(z) .
$$

Substituting (4.4) into (4.7) and (4.7) into (2.10), we obtain

$$
\begin{aligned}
H(\lambda)= & \left(I_{p}, O_{p \times q}\right)\left(\theta_{11}(\lambda) \sigma(\lambda)+\theta_{12}(\lambda)\right) \\
& \times\left\{\left[a(\lambda) \theta_{21}(\lambda)-b(\lambda)\left(O_{q \times p}, I_{q}\right) \theta_{11}(\lambda)\right] \sigma(\lambda)\right. \\
& \left.+a(\lambda) \theta_{22}(\lambda)-b(\lambda)\left(O_{q \times p}, I_{q}\right) \theta_{12}(\lambda)\right\}^{-1} \\
= & \left(\psi_{11}(\lambda) \sigma(\lambda)+\psi_{12}(\lambda)\right)\left(\psi_{21}(\lambda) \sigma(\lambda)+\psi_{22}(\lambda)\right)^{-1}
\end{aligned}
$$

where

$$
\Psi(\lambda)=\left(\begin{array}{ll}
\psi_{11}(\lambda) & \psi_{12}(\lambda) \\
\psi_{21}(\lambda) & \psi_{22}(\lambda)
\end{array}\right)=\left(\begin{array}{ccc}
I_{p} & 0 & 0 \\
0 & -b(\lambda) I_{q} & a(\lambda) I_{q}
\end{array}\right) \Theta(\lambda) .
$$

Substituting (4.1) into (4.8) and using (3.14) we come to (4.5) which ends the proof of the theorem

Remark 4.3: The description (4.6) follows directly from the result that the function $H$ is a solution to problem $I\left(\mathbf{H}_{p}^{p \times q}\left(I_{q}\right)\right)$ if and only if the kernel

$$
\mathbf{K}_{H}(\lambda, \omega)=\left(\begin{array}{cc}
K & \left(a(\omega) A^{*}-b(\omega) B^{*}\right)^{-*} M J V(\omega)^{*} \\
V(\lambda) J M^{*}\left(a(\lambda) A^{*}-b(\lambda) B^{*}\right)^{-1} & \frac{V(\lambda) J V(\omega)^{*}}{\rho_{\omega}(\lambda)}
\end{array}\right)
$$

is non-negative on $\Omega_{+}$, where $V(\lambda)=\left(I_{p}, b(\lambda) H(\lambda), a(\lambda) H(\lambda)\right)$ and $A, B$ and $M$ are matrices given by (3.13) and (3.14). This fact can be proved similarly to Theorem 3.4. 


\section{References}

[1] Alpay, D. and V. Bolotnikov: Two-sided Nevanlinna-Pick interpolation for a class of matrix-valued functions. Z. Anal. Anw. 12 (1993), $211-238$.

[2] Alpay, D., Bolotnikov, V. and Y. Peretz: The Nevanlinna-Pick $\mathrm{H}_{2}$ interpolation problem. Proc. Amer. Math. Soc. (to appear).

[3] Alpay, D. and H. Dym: On reproducing kernel spaces, the Schur algorithm and interpolation in a general class of domains. Oper. Theory: Adv. Appl. 59 (1992), 30 . 77.

[4] Alpay, D. and H. Dym: On a new class of reproducing kernel spaces and a new generalization of the Iohvidov laws. Lin. Alg. Appl. 178 (1993), 109 - 183.

[5] Alpay, D. and H. Dym: On a new class of structured reproducing kernel Hilbert spaces. J. Funct. Anal. 111 (1993), 1 - 28.

[6] Ball, J., Gohberg, I. and L. Rodman: Interpolation of Rational Matrix Functions. Oper. Theory: Adv. Appl. 45 (1990), 1 - 605.

[7] Bolotnikov, V.: Carathéodory-Féjer problem on pairs of meromorphic functions. Preprint. Beer Sheva (Israel): Ben Gurion Univ. of the Negev/Dep. Math. 1994.

[8] Dubovoi, V., Fritzsche, B., and B. Kirstein: Matricial Version of the Classical Schur Problem (Teubner - Texte zur Mathematik: Vol. 129). Leipzig: B. G. Teubner Verlagsges. 1992.

[9] Duren, P. L. and D. L. Williams: Interpolation problems in function spaces. J. Funct. Anal. 9 (1972), 75 - 86.

[10] Hoffman, K.: Banach Spaces of Analytic Functions. Englewood Cliffs (N. J.): Prentice Hall 1962.

[11] Kovalishina, I.: Analytic theory of a class of interpolation problems. Math. USSR Izv. 22 (1984), $419-463$.

[12] Kovalishina, I. and V. Potapov: An indefinite metric in the Nevanlinna-Pick problem. Amer. Math. Soc. Transl. (2) 138 (1988), 15 - 19.

[13] Kovalishina, I. and V. Potapov: The radii of a Weyl disk in the matrix Nevanlinna-Pick problem. Amer. Math. Soc. Transl. (2) 138 (1988), 37 - 54.

[14] Sarason, D.: Nearly invariant subspaces of the backward shift. Oper. Theory: Adv. Appl. 35 (1988), 481 - 493.

[15] Sarason, D.: Exposed points in $H^{1}$. Part I. Oper. Theory: Adv. Appl. 41 (1989), 485 496.

[16] Sarason, D.: Exposed points in $H^{1}$. Part II. Oper. Theory: Adv. Appl. 48 (1989), 333 347. 